\title{
CARACTERIZACIÓN FÍSICO-QUÍMICA DE LOS SEDIMENTOS DEL HUMEDAL LAgUNA BELla EN LA SELVA DE HUÁNUCO, PERÚ
}

\section{PHYSICOCHEMICAL CHARACTERIZATION OF THE SEDIMENTS OF THE LAGUNA BELLA WETLAND IN THE JUNGLE OF HUÁNUCO, PERU}

\author{
Lauriano Zavaleta De la Cruz ${ }^{1}$, Manuel Ñique Alvarez² y José Lévano Crisóstomo³
}

\begin{abstract}
Resumen
Las lagunas en la Amazonia están siendo impactadas por las actividades agrícolas y la información sobre sus sedimentos es escasa; por lo que el objetivo del estudio fue determinar las características físico-químicas de los sedimentos del humedal Laguna Bella, lo que contribuirá al conocimiento de su calidad ambiental. Así, mediante técnicas estandarizadas se analizaron los sedimentos colectados en seis puntos de muestreo. Los resultados muestran los valores de la densidad, conductividad, $\mathrm{pH}$, materia orgánica, $\mathrm{N}, \mathrm{P}, \mathrm{Pb}, \mathrm{Cu}, \mathrm{Fe}, \mathrm{Mn}, \mathrm{Zn}$ y K, los que se compararon con cinco normas internacionales. Considerando la norma Ontario, la materia orgánica es menor al Nivel de efecto severo, $\mathrm{N}$ está entre el Nivel de efecto más bajo y severo, mientras que $\mathrm{P}$ es inferior a la concentración de Nivel de efecto más bajo; $\mathrm{Pb}$ está a un nivel moderadamente contaminado, según la norma USEPA, pero supera a la norma NOAA. El Cu superó el nivel máximo permisible de la norma NOAA; Fe supera el nivel máximo permisible por las normas USEPA, Ontario y NOAA; Mn supera el nivel máximo permisible de las normas NOAA y Zn supera la norma NOAA. Se concluye que, la densidad de los sedimentos es uniforme, el $\mathrm{pH}$ ligeramente alcalino, la salinidad es despreciable y la concentración de metales como el $\mathrm{Fe}>\mathrm{Mn}>\mathrm{Zn}>\mathrm{Pb}>\mathrm{Cu}$, superan la normatividad internacional e indica el deterioro de la calidad ambiental del humedal.
\end{abstract}

Palabras clave: humedal, meandro, sedimentos, Amazonia, metales, contaminantes.

\begin{abstract}
The lagoons in the Amazon are being impacted by agricultural activities and the information about their sediments is scarce; therefore, the objective of the study was to determine the physicochemical characteristics of the sediments of the Laguna Bella, wetland which will contribute to the knowledge of its environmental quality. Thus, using standardized techniques, sediments collected at six sampling points were analyzed. The results show the values of density, organic matter, conductivity, $\mathrm{pH}$, organic matter, $\mathrm{N}, \mathrm{P}, \mathrm{Pb}, \mathrm{Cu}, \mathrm{Fe}, \mathrm{Mn}, \mathrm{Zn}$ and $\mathrm{K}$, which were compared with five international standards. Considering the Ontario standard, organic matter is below the severe effect level; $\mathrm{N}$ is between the lowest and most severe effect level, while $\mathrm{P}$ is below the lowest effect level concentration; $\mathrm{Pb}$ is at a moderately contaminated level, according to the USEPA standard, but exceeds the NOAA standard. $\mathrm{Cu}$ exceeded the maximum permissible level of the NOAA standard; $\mathrm{Fe}$ exceeds the maximum permissible level of USEPA, Ontario, and NOAA standards; Mn exceeds the maximum allowable level of NOAA standards, and $\mathrm{Zn}$ exceeds the NOAA standard. It is concluded that the density of the sediments is uniform, the $\mathrm{pH}$ is slightly alkaline, the salinity is negligible, and the concentration of metals, such as $\mathrm{Fe}>\mathrm{Mn}>\mathrm{Zn}>\mathrm{Pb}>\mathrm{Cu}$, exceeds international standards and indicates the deterioration of the environmental quality of the wetland.
\end{abstract}

Key words: wetland, meander, sediments, Amazon, metals, pollutants.

\section{Introducción}

Los sedimentos de los lagos constituyen archivos de procesos ambientales (Poerschmann et al., 2017) y los indicadores físicos, químicos y biológicos que se preservan en los sedimentos lacustres proporcionan información sobre eventos pasados y el efecto de los mismos en el sistema lacustre (Cuña, 2018); lo que permite inferir la distribución espacial de algunos contaminantes, la estructura de poblaciones bacterianas y bentónicas, fitolitos, metales pesados y otros componentes que son estudiados por la paleolimnología (Arias \& Ramírez, 2009).
Los sedimentos, al ser muy sensibles a los cambios en el ambiente, retienen evidencia de procesos de deposición de materiales derivados de factores exógenos como el clima, la erosión, la meteorización y la evolución de la flora y fauna; y endógenas como la tectónica, la actividad volcánica, las fluctuaciones geomagnéticas y otros (Herrera, 2011; Xu et al., 2017); asimismo, los depósitos de sedimentos en los lagos funcionan bien sea como fuente o como reserva de muchos de los nutrientes (Ramírez \& Noreña, 2004), por lo que sus estudios deben ser incluidos en la caracterización de los sedimentos de humedales. 
Julio - Diciembre 2021

Los parámetros característicos que se analizan con mayor frecuencia en los sedimentos son $\mathrm{pH}$, conductividad eléctrica, materia orgánica, $\mathrm{N}, \mathrm{P}, \mathrm{K}, \mathrm{Na}$, $\mathrm{Ca}, \mathrm{Cu}, \mathrm{Mn}, \mathrm{Zn}$ y metales como el As, Cd, Cr, $\mathrm{Hg}$ y $\mathrm{Pb}$, que son indicadores de contaminación conforme lo indican González et al. (2018), Santana (2020) y Villalba (2020).

En ese sentido, el humedal Laguna Bella, localizado en la selva de Huánuco, Perú, es parte de los humedales distribuidos en la región Amazónica. Presenta variedad de recursos bióticos y potencial turístico; sin embargo, está siendo afectada por diversos tensores ambientales (Castillo, 2018), principalmente por la intensa actividad agrícola de la zona, sin disponer de información sobre la calidad de sus sedimentos. En ese sentido, el presente estudio tuvo por objetivo caracterizar las propiedades físico-químicas de los sedimentos del humedal Laguna Bella, lo que contribuirá al conocimiento de su calidad ambiental.

\section{Materiales y métodos}

Área de estudio

El humedal Laguna Bella es un cuerpo de agua somero y geomorfológicamente es un meandro abandonado, conocido localmente como "tipishca", que se formó cuando el río Huallaga cortó el cuello del meandro acortando su curso, lo que hizo que el antiguo canal quede rápidamente bloqueado y luego quede separado del cauce, conforme lo explica Luque (2018).

El humedal se localiza a un kilómetro del río Huallaga y en sus alrededores presenta vegetación secundaria y cultivos agrícolas y le corresponde las siguientes coordenadas UTM: Este 352312 y Norte 9064722. Políticamente pertenece al Centro Poblado Paraíso, Distrito de Cholón, Provincia de Marañón, Región Huánuco, Perú. El humedal posee 1.24 ha, con perímetro de $2348.76 \mathrm{~m}$ y a una elevación de 503 msnm. Presenta los siguientes datos climáticos medios anuales: temperatura mínima de $21.0^{\circ} \mathrm{C}$, temperatura máxima de $29.7^{\circ} \mathrm{C}$ y precipitación de $2574.8 \mathrm{~mm}$ (GOREHCO, 2016).

Puntos de Muestreo

Utilizando una lancha se definieron seis puntos de muestreo, los que se muestran en la Figura 1, así como las respectivas profundidades. Los muestreos se realizaron en noviembre de 2016.

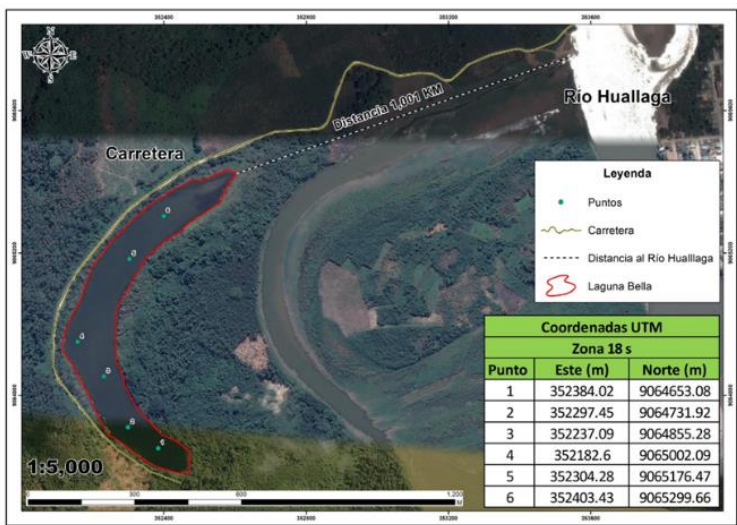

Figura 1. Puntos de muestreo en humedal Laguna Bella (16/11/ 2016).

Muestreo y pretratamiento de las muestras de sedimentos

Las muestras de sedimentos se colectaron según Andreu \& Camacho (2002) y lo recolectado (3 Kg aproximadamente) se depositó en recipientes de plástico con tapa hermética, se preservaron con hielo embolsado dentro de una caja de Tecnopor y luego fueron trasladados al Laboratorio de Química de la Universidad Nacional Agraria de la Selva, donde se almacenaron bajo refrigeración a $4{ }^{\circ} \mathrm{C}$.

Determinación de las características físico-químicas de $\underline{\text { los sedimentos }}$

Los procedimientos para el análisis físico-químico de los sedimentos se resumen en la Tabla 1.

Tabla 1. Métodos analíticos empleados en los análisis físicos y químicos de las muestras de sedimentos.

\begin{tabular}{|c|c|}
\hline Parámetro & Método \\
\hline Humedad $\left(\% \mathrm{H}_{2} \mathrm{O}\right)$ & $\begin{array}{l}\text { Gravimétrico: (Zagal \& Sadzawka, } \\
\text { 2007) }\end{array}$ \\
\hline $\begin{array}{l}\text { Materia orgánica } \\
(\% \mathrm{MO})\end{array}$ & $\begin{array}{l}\text { Incineración: (Bernal \& Betancur, } \\
\text { 1996) }\end{array}$ \\
\hline $\mathrm{pH}$ & $\begin{array}{l}\text { Potenciométrico (Andrades et al., } \\
\text { 2015) }\end{array}$ \\
\hline $\begin{array}{l}\text { Conductividad } \\
(\mu \mathrm{S} / \mathrm{cm})\end{array}$ & $\begin{array}{l}\text { Potenciométrico (Andrades et al., } \\
\text { 2015) }\end{array}$ \\
\hline $\begin{array}{l}\text { Densidad } \quad \text { real } \\
\left(\mathrm{g} / \mathrm{cm}^{3}\right)\end{array}$ & $\begin{array}{l}\text { Picnómetro en agua (Villaseñor, } \\
\text { 2015). }\end{array}$ \\
\hline $\begin{array}{l}\text { Nitrógeno total (\% } \\
\text { N) }\end{array}$ & $\begin{array}{l}\text { Microkjeldahl (Fernández et al., } \\
\text { 2006). }\end{array}$ \\
\hline $\begin{array}{l}\text { Fósforo disponible } \\
(\mathrm{mg} / \mathrm{Kg})\end{array}$ & Olsen (Gilabert et al., 2015) \\
\hline
\end{tabular}

Determinación del contenido de elementos metálicos en los sedimentos

Los métodos analíticos empleados para determinar el contenido de elementos metálicos se indican en la Tabla 2. 
Tabla 2. Métodos analíticos empleados en la determinación de elementos metálicos en sedimentos.

\begin{tabular}{|c|c|}
\hline $\begin{array}{l}\text { Parámetro / } \\
\text { Unidades }\end{array}$ & Método y Descripción \\
\hline $\begin{array}{l}\text { Concentración } \\
\text { de metales } \\
\mathrm{mg} / \mathrm{Kg}\end{array}$ & $\begin{array}{l}\text { Digestión ácida fuerte. } \\
\text { - Digestión con ácido nítrico y ácido } \\
\text { clorhídrico. } \\
\text { Espectrofotometría de Absorción } \\
\text { Atómica (Method 3050B) (USEPA, } \\
\text { 1996). } \\
\text { - Medición espectrofotométrica de } \\
\text { absorción atómica con llama. } \\
\text { - Fuentes de radiación: Lámparas de } \\
\text { cátodo hueco (LCH). } \\
\text { - Técnica: Curva estándar. }\end{array}$ \\
\hline
\end{tabular}

Asimismo, al no contar el Perú con Estándares Nacionales de Calidad Ambiental para contenido de metales en sedimentos, se consideraron normas ambientales de Canadá, México y Estados Unidos, los que se resumen en la Tabla 3.

\section{Resultados \\ Características físico-químicas de los sedimentos}

Los resultados del análisis físico-químico de los sedimentos de los puntos de muestreo $\left(\mathrm{P}_{\mathrm{n}}\right)$ se presentan en la Tabla 4. Según ésta, el mayor contenido de humedad promedio se obtuvo en el $\mathrm{P}_{6}(67.43 \%)$, mientras que el menor se determinó en el $\mathrm{P}_{5}(59.70 \%)$; el mayor porcentaje de materia orgánica se encontró en el $\mathrm{P}_{1}(12.05 \%)$ y el menor en el $\mathrm{P}_{5}(11.26 \%)$. Respecto al $\mathrm{pH}$, los valores son bastante homogéneos. El mayor valor se obtuvo en el $\mathrm{P}_{5}(7.98)$ y el menor en el $\mathrm{P}_{6}$ (7.67). La conductividad eléctrica de los sedimentos osciló entre 188.14 y $269.72 \mu \mathrm{S} / \mathrm{cm}$. La mayor densidad promedio se observó en el $\mathrm{P}_{5}$, con un valor de 2.65 $\mathrm{g} / \mathrm{cm}^{3}$, mientras que el menor promedio se obtuvo en el $\mathrm{P}_{1}$, con una media de $2.45 \mathrm{~g} / \mathrm{cm}^{3}$. El mayor contenido promedio de $\mathrm{N}$ se obtuvo en el $\mathrm{P}_{4}$, con un valor de $0.282 \%$, mientras que el menor promedio se obtuvo en el $\mathrm{P}_{1}$, con una media de $0.204 \%$. El mayor contenido promedio de $\mathrm{P}$ se obtuvo en el $\mathrm{P}_{1}$, con un valor de $48.455 \%$, mientras que el menor valor promedio se obtuvo en el $\mathrm{P}_{6}$, con una media de $25.081 \%$.

Contenido de elementos metálicos en los sedimentos

Los valores del contenido de elementos metálicos se muestran en el Tabla 5. Según ésta, el mayor contenido de $\mathrm{Pb}$ promedio se encontró en el $\mathrm{P}_{2}$ $(63.54 \mathrm{mg} / \mathrm{Kg})$, mientras que el menor en el $\mathrm{P}_{6}$ con $56.85 \mathrm{mg} / \mathrm{Kg}$. La mayor concentración promedio de $\mathrm{Cu}$ se encontró en el $\mathrm{P}_{2}$, con $35.71 \mathrm{mg} / \mathrm{Kg}$, mientras que la menor concentración se determinó en el $\mathrm{P}_{1}$ $(34.60 \mathrm{mg} / \mathrm{Kg})$. La mayor concentración de $\mathrm{Fe}$ se encontró en el $\mathrm{P}_{6}(44759.46 \mathrm{mg} / \mathrm{Kg})$ y la menor concentración en el $\mathrm{P}_{1}(34900.58 \mathrm{mg} / \mathrm{Kg})$. El mayor contenido de $\mathrm{Mn}$ se obtuvo en el $\mathrm{P}_{4}(918.29 \mathrm{mg} / \mathrm{Kg})$, mientras que el menor contenido en el $\mathrm{P}_{1}$ (678.74 mg/Kg). La mayor concentración promedio de $\mathrm{Zn}$ se obtuvo en el $\mathrm{P}_{4}(86.14 \mathrm{mg} / \mathrm{Kg})$, mientras que la menor se obtuvo en el $\mathrm{P}_{1}$, con $69.43 \mathrm{mg} / \mathrm{Kg}$. Asimismo, el mayor contenido de $\mathrm{K}$ se determinó en el $\mathrm{P}_{5}$ (5 $190.82 \mathrm{mg} / \mathrm{Kg}$ ), mientras que el menor contenido se encontró en el $\mathrm{P}_{1}$ (2 $\left.326.73 \mathrm{mg} / \mathrm{Kg}\right)$.

\section{Discusión}

El porcentaje de humedad promedio total $(63.28 \%)$ que se muestra en la Tabla 4 es ligeramente superior a lo reportado para la laguna Limoncocha-Ecuador $(60.96 \%)$ determinado por Ordoñez (2013).

El contenido de materia orgánica (MO) promedio, indicado en la Tabla $4(11.77 \%)$, es superior a lo encontrado por De La Mora-Orozco et al. (2018) en los sedimentos de una presa $(9.61 \%)$ y muy superior al valor encontrado en sedimentos de la laguna "La Zacatecana" en México que, según Covarrubias et al. (2018), está en el rango de 4.3 - 1.2\% . Las fuentes de materia orgánica sedimentaria pueden dividirse en endógenas y exógenas. La primera proviene principalmente de bacterias lacustres, algas, plantas acuáticas y otros grandes organismos acuáticos, y la segunda proviene principalmente de plantas terrestres de alto grado y de actividades humanas (Xu et al., 2017).

Los valores de $\mathrm{pH}$, mostrados en la Tabla 4 , se ubican en el rango de neutros y medianamente alcalinos (6.6 hasta 8.5), de acuerdo a la Norma Oficial Mexicana NOM-021-RECNAT-2000 (Secretaria de Gobernación, 2002), De La Mora-Orozco et al. (2018) y Covarrubias et al. (2018).

La conductividad eléctrica osciló entre 188.14 y $269.72 \mu \mathrm{S} / \mathrm{cm}$, valores que corresponden a efectos despreciables de salinidad, según la Norma Oficial Mexicana NOM-021 RECNAT-2000 (Secretaria de Gobernación, 2002). De igual manera, son muy inferiores a los valores determinados por De La MoraOrozco et al. (2018), que reportaron $2100 \mu \mathrm{S} / \mathrm{cm}$.

Los sedimentos del humedal Laguna Bella, considerados en la Tabla 4, presentan una densidad media de $2.57 \mathrm{~g} / \mathrm{cm}^{3}$, que es menor a lo determinado por Carbajal et al. (2018) para la laguna del Golfo de California $\left(2.65 \mathrm{~g} / \mathrm{cm}^{3}\right)$.

La concentración total promedio de $\mathrm{N}$ en los sedimentos del humedal Laguna Bella (0.22\%), indicado en la Tabla 4, está comprendida entre el Nivel de efecto más bajo $(0.055 \%)$ y el Nivel de efecto severo $(0.480 \%)$ estipulado por la norma de Ontario (Persaud et al., 1993). Es muy inferior a la concentración media de $\mathrm{N}$ total para Bahía Oriental del Lago LácarArgentina $(3.91 \%)$ reportado por Temporetti et al. (2014) pero mayor a lo determinado por Liu et al. (2021) en el lago Balkhash $(0.16 \%)$ y Ji et al. (2019) para el lago Baiyangdian (0.000216\%), ambos en Asia.

La concentración total promedio de $\mathrm{P}$ en los sedimentos del humedal Laguna Bella (35.42 ppm) reportada en la Tabla 4, es aproximadamente 17 veces menor para el Nivel de efecto más bajo (600 ppm) estipulado por la norma Ontario (Persaud et al., 1993) 
Julio - Diciembre 2021

e inferior al rango 900 - $380 \mathrm{mg} / \mathrm{Kg}$ reportado por Varol (2020) para la presa Keban en Turquía, pero mayor al valor registrado para el lago Baiyangdian $(0.72 \mathrm{mg} / \mathrm{Kg})$ por Ji et al. (2019) y De La Mora-Orozco et al. (2018) que reportaron $27.10 \mathrm{mg} / \mathrm{Kg}$ para un ecosistema lacustre.

La concentración promedio de $\mathrm{Pb}(59.78 \mathrm{mg} / \mathrm{Kg})$, mostrado en la Tabla 5, se encuentra en el rango de moderadamente contaminado (40 - $60 \mathrm{mg} / \mathrm{Kg})$, según USEPA (1993), pero es inferior a lo estipulado por la SEMARNAT (2007) (Tabla 3); y de acuerdo a la guía de calidad ambiental canadiense para sedimentos (CCME, 2001), las concentraciones de $\mathrm{Pb}$ se ubican en el rango de la Guía provisional de sedimento (ISQG) (35 mg/Kg) y el Nivel de efecto probable (PEL) (91.3 $\mathrm{mg} / \mathrm{Kg}$ ). Con referencia a la norma NOAA (NOAA, 2008), es aproximadamente 3.5 veces superiores (4 - 17 mg/Kg) y, según la norma Ontario (Persau et al., 1993), está entre el Nivel de efecto más bajo (LEL) y el Nivel de efecto severo (SEL), (31-250 $\mathrm{mg} / \mathrm{Kg}$, respectivamente).

Asimismo, la concentración de $\mathrm{Pb}$ en el presente estudio, fue superior a lo encontrado por Ji et al. (2019) en el lago Baiyangdian en China $(44.16 \mathrm{mg} / \mathrm{Kg})$, pero inferior a los registrado por Babcsányi et al. (2020) que indica un rango de 62 - $72 \mathrm{mg} / \mathrm{Kg}$ para sedimentos de meandros abandonados en el valle del rio Tisza, Hungría.

Según los estándares establecidos para sedimentos por las normas indicadas en la Tabla 3, la concentración de $\mathrm{Cu}$ encontrada en los sedimentos del humedal Laguna Bella $(35.24 \mathrm{mg} / \mathrm{Kg})$ indica que están catalogados como de contaminación moderada según la USEPA (1993) (25-50 mg/Kg), similar al nivel de ISQG que indica el valor de $35.7 \mathrm{mg} / \mathrm{Kg}$ según CCME (2001), pero superior a lo estipulado por la NOAA (2008), y está entre el Nivel de efecto más bajo y Nivel de efecto severo según la norma Ontario (Persaud et al., 1993), (16-110 mg/Kg).

Además, las concentraciones de $\mathrm{Cu}$ encontradas en el presente estudio son mayores a los de_Laguna de Bustillos, en Chihuahua, México $(3.5 \mathrm{mg} / \mathrm{Kg}$ ) según Rubio-Arias et al. (2018), pero menor a lo_indicado por Ji et al. (2019) lago Baiyangdian en China (39.20 $\mathrm{mg} / \mathrm{Kg}$ ) y Babcsányi et al. (2020) que reporta un rango de $58-82 \mathrm{mg} / \mathrm{Kg}$.

Los sedimentos del humedal en estudio (Tabla 5) tienen un nivel alto de $\mathrm{Fe}(40430.57 \mathrm{mg} / \mathrm{Kg})$ debido a la intensa meteorización de los suelos de selva que son suelos ácidos muy ricos en óxidos e hidróxidos de Fe y Mn, por el intenso lavaje de las aguas de lluvia, y superan a la norma USEPA-823-B93-001 indicada en USEPA (1993) (> $25000 \mathrm{mg} / \mathrm{Kg}$ ), presentan una concentración promedio muy superior a lo estipulado por la norma NOAA (9 900 - $18000 \mathrm{mg} / \mathrm{Kg}$ ) (NOAA, 2008), y muy similar a la concentración del Nivel de efecto severo de la norma Ontario (Persaud et al., 1993).
Igualmente, los valores del Fe considerados en la Tabla 5, presentaron concentraciones muy superiores a lo reportado Rubio-Arias et al. (2018) para la Laguna de Bustillos, Chihuahua, México (rango: 3174 - 3315 $\mathrm{mg} / \mathrm{Kg}$ ) y Portz et al. (2020) para la laguna Mallorquin en Barranquilla, Colombia (14 269.8 - 11993.9 $\mathrm{mg} / \mathrm{Kg}$ ).

Las concentraciones de $\mathrm{Mn}$ en los sedimentos promedio $(839.10 \mathrm{mg} / \mathrm{Kg})$, indicadas en la Tabla 5, superaron al doble de la concentración estipulada por la norma NOAA (400 mg/Kg) (NOAA, 2008), pero se encuentran entre el Nivel de efecto más bajo y el Nivel de efecto severo $(460-1100 \mathrm{mg} / \mathrm{Kg})$ de la norma Ontario (Persaud et al., 1993). Del mismo modo, es superior a lo reportado por Cabrera (2018) en el lago Limoncocha, Ecuador (218 mg/Kg) y Ji et al. (2019) que encontró $506.46 \mathrm{mg} / \mathrm{Kg}$ en el lago Baiyangdian.

Los sedimentos del humedal Laguna Bella (Tabla 5) presentan una nula contaminación con $\mathrm{Zn}$ (79.2 $\mathrm{mg} / \mathrm{Kg}$ ), según USEPA (1993), indicado en la Tabla 3; son inferiores a lo indicado por la ISQG (123 $\mathrm{mg} / \mathrm{Kg}$ ), según CCME (2001). Así mismo, es inferior al Nivel de efecto más bajo estipulado por la norma Ontario (Persaud et al., 1993), cuyo valor es de 120 $\mathrm{mg} / \mathrm{Kg}$; sin embargo, es muy superior a lo estipulado en la norma NOAA (7-38 mg/Kg) (NOAA, 2008). Y comparando con resultados de estudios similares de $\mathrm{Zn}$, mostrados en la Tabla 5, el humedal Laguna Bella presentó una concentración menor a lo determinado por Babcsányi et al. (2020) que reportaron un rango de 290 - $215 \mathrm{mg} / \mathrm{Kg}$, pero mayor a lo reportado por Cabrera (2018) en el lago Limoncocha, Ecuador $(29.3 \mathrm{mg} / \mathrm{Kg})$ y Moreno et al. (2018) para el lago Titicaca (9.19 - 6.35 $\mathrm{mg} / \mathrm{Kg}$ ).

La concentración de K (4 $052.64 \mathrm{mg} / \mathrm{Kg})$ en los sedimentos del humedal Laguna Bella (Tabla 5) superó a lo reportado por Tendaupenyu \& Magadza (2019) en el Lake Chivero, Zimbabwe (2 $511.32 \mathrm{mg} / \mathrm{Kg}$ ) y lo encontrado por De La Mora-Orozco (2018) en los sedimentos de la presa La Vegas en Jalisco, México (731.58 mg/Kg).

\section{Conclusiones}

La densidad de los sedimentos es uniforme en todos los puntos de muestreo, el valor del pH está en el rango de ligeramente alcalino y el valor de la conductividad tiene efecto despreciable de salinidad.

El contenido de materia orgánica es menor al Nivel de Efecto Severo estipulado por la norma Ontario.

Las características químicas de los sedimentos del humedal Laguna Bella indican el deterioro de su calidad ambiental al presentar valores de Fe que superan los límites permisibles de las normas USEPA, Ontario y NOAA, y de $\mathrm{Pb}, \mathrm{Cu}, \mathrm{Zn}$ y $\mathrm{Mn}$ a la norma NOAA. 


\section{Literatura citada}

Andrades M., Moliner A. \& Masaguer A. 2015. Prácticas de edafología. Métodos didácticos para análisis de suelos. Material didáctico - Agricultura y alimentación №15. Servicio de Publicaciones / Universidad de La Rioja. https://dialnet.unirioja.es/descarga/libro/580696.pdf.

Andreu E. \& Camacho A. 2002. Recomendaciones para la toma de muestras de agua, biota y sedimentos en humedales Ramsar. Ministerio de Medio Ambiente. España.

http://www.sehumed.es/banco/archivos/recomendacione S_es.zip.

Arias J. \& Ramírez J. 2009. Caracterización preliminar de los sedimentos de un embalse tropical: represa $\mathrm{La} \mathrm{Fe}(\mathrm{El}$ Retiro, Antioquia, Colombia). Limnetica, 28(1): 65-78. https://www.limnetica.com/documentos/limnetica/limne tica-28-1-p-65.pdf.

Babcsányi I., Tamás M., Szatmári J. \& Hambek-Oláh B. 2020. Assessing the impacts of the main river and anthropogenic use on the degree of metal contamination of oxbow lake sediments (Tisza River Valley, Hungary). Journal of Soils and Sediments, 20: 1662-1675. https://doi.org/10.1007/s11368-019-02516-y.

Bernal G. \& Betancur J. 1996. Sedimentología de lagunas costeras: ciénaga grande de Santa Marta y ciénaga de Pajarales. Bol. Invest. Mar. Cost., 25: 49-75. https://doi.org/10.25268/bimc.invemar.1996.25.0.370

Cabrera J. 2018. Evaluación de la contaminación por metales pesados en sedimentos y suelos de la Reserva Biológica Limoncocha - Ecuador mediante índices de polución. Tesis Master Universitario en Investigación en Ingeniería Industrial. Universidad de Cantabria. España. URI: http://hdl.handle.net/10902/14196.

Carbajal N., Montaño Y., Páez F. \& Guevara M. 2018. Sediment dynamics in altata ensenada del pabellón, a coastal lagoon located in the Gulf of California. Journal of Coastal Conservation, 22: 709-720. https://doi.org/10.1007/s11852-018-0603-3.

Castillo F. 2018. Evaluación de la calidad ambiental del Humedal Refugio de Vida Silvestre Sistema Lagunar de Tísma, Masaya, Nicaragua. Tesis de Maestría, Universidad Nacional Agraria. Nicaragua. https://repositorio.una.edu.ni/3682/.

CCME. 2001. Canadian sediment quality guidelines for the protection of aquatic life: Summary tables. Updated. In: CCME (Canadian Council of Ministers of the Environment) Canadian Environmental Quality Guidelines.

https://www.elaw.org/system/files/sediment_summary_t able.pdf

Covarrubias S., Flores de la Torre J., Maldonado M., Avelar F. \& Peña J. 2018. Spatial Variability of Heavy Metals in Soils and Sediments of "La Zacatecana" Lagoon, Mexico. Applied and Environmental Soil Science, 2018: 9612412. https://doi.org/10.1155/2018/9612412.

Cuña C. 2018. Diversidad y morfometría diatomológica frente a variaciones ambientales: estudio de registros actuales y paleolimnológicos holocenos de lagos someros del sudeste de Sudamérica. Tesis doctoral Universidad Nacional de Córdoba. Argentina. URI: http://hdl.handle.net/11336/88744.
De La Mora-Orozco C., García-Hernández S.B., GonzálezAcuña I.J., Flores-López H.E., Martínez-Orozco E. \& Chávez-Durán A.A. 2018. Caracterización química de sedimentos en un ecosistema lacustre. En: Memoria del XLIII Congreso Nacional de la Ciencia del Suelo; Buenavista, Saltillo, Coahuila, México 1 a 5 de octubre de 2018. 244-249. SMCS (Sociedad Mexicana de la Ciencia del Suelo. México. https://www.researchgate.net/publication/333392821_C ARACTERIZACION_QUIMICA_DE_SEDIMENTOS _EN_UN_ECOSISTEMA_LACUSTRE.

Fernández L., Rojas N., Roldán T., Ramírez M., Zegarra H., Uribe R., Reyes R., Flores D. \& Arce J. 2006. Manual de técnicas de análisis de suelos aplicadas a la remediación de sitios contaminados. Instituto Mexicano del Petróleo. Secretaría de Medio Ambiente y Recursos Naturales. México.

https://biblioteca.semarnat.gob.mx/janium/Documentos/ Ciga/Libros2011/CG008215.pdf.

Gilabert J., Arrieche I., León M. \& López I. (Compiladores). 2015. Análisis de suelos para diagnóstico de fertilidad. Manual de métodos y procedimientos de referencia. Instituto Nacional de Investigaciones Agrícolas \Centro Nacional de Investigaciones Agropecuarias. Venezuela. https://www.researchgate.net/publication/323999788_A nalisis_de_suelo_para_el_diagnostico_de_fertilidad_ma nual_de_metodos_y_procedimientos_de_referencia

González V., Valle S., Nirchio M., Olivero J., Tejeda L., Valdelamar J., Pesantes F. \& González K. 2018. Evaluación del riesgo de contaminación por metales pesados $(\mathrm{Hg}$ y $\mathrm{Pb})$ en sedimentos marinos del Estero Huaylá, Puerto Bolívar, Ecuador. Revista del Instituto de Investigación de la Facultad de Minas, Metalurgia y Ciencias $\quad$ Geográficas, 21(41): 75-82. DOI: 10.15381/iigeo.v21i41.14995.

GOREHCO. 2016. Zonificación Ecológica y Económica, Estudio Climático, Provincia de Marañón. GOREHCO (Gobierno Regional de Huánuco). Perú. http://zee.regionhuanuco.gob.pe/wpcontent/uploads/2016/08/mem-Clima_Maranon.pdf.

Herrera D. 2011. Estratigrafía y análisis de facies de los sedimentos lacustres del Cuaternario tardío de la cuenca de Chalco, México [tesis de maestría, Universidad Nacional Autónoma de México]. Repositorio UNAM. http://132.248.9.195/ptb2011/marzo/0667612/Index.htm 1

Ji Z., Zhang Y., Zhang H., Huang C. \& Pei Y. 2019. Fraction spatial distributions and ecological risk assessment of heavy metals in the sediments of Baiyangdian Lake, Ecotoxicology and Environmental Safety, 174: 417-428. https://doi.org/10.1016/j.ecoenv.2019.02.062

Liu W., Ma, L., Abuduwaili J., Issanova G. \& Saparov G. 2021. Sediment Organic Carbon Sequestration of Balkhash Lake in Central Asia. Sustainability, 13(17), 9958. https://doi.org/10.3390/su13179958

Luque G. 2018. Peligro por inundación y erosión fluvial en las localidades de San Carlos y Nuevo Olaya (Contamana) y localidad de Samán (Sarayacu), provincia Ucayali, departamento Loreto. Informe Técnico $\mathrm{N}^{\circ}$ A6814. INGEMMET (Instituto Geológico, Minero y Metalúrgico).

Perú. URI: https://repositorio.ingemmet.gob.pe/handle/20.500. $12544 / 1685$. 
Moreno E., Argota G., Alfaro R., Aparicio M., Atencio S. \& Goyzueta G. 2018. Cuantificación de metales en sedimentos superficiales de la bahía interior, lago Titicaca (Perú). Revista de Investigaciones Altoandinas, 20(1): 9-18. https://doi.org/10.18271/ria.2018.326.

NOAA. 2008. Screening Quick Reference Tables. OR\&R Report 08-1. NOAA (National Oceanic and Atmospheric Administration). USA. https://repository.library.noaa.gov/view/noaa/9327.

Ordoñez C. 2013. Caracterización geoquímica de sedimentos de la laguna Limoncocha. Tesis de Magister en Gestión Ambiental en la Industria, Universidad Internacional Sek. https://repositorio.uisek.edu.ec/bitstream/123456789/61 0/1/CARLOS\%20GILBERTO\%20ORDO\%C3\%91EZ $\%$ 20CAMPAIN.pdf.

Persaud D., Jaagumagi R. \& Hayton A. 1993. Guidelines for the protection and management of aquatic sediment quality in Ontario. Ministry of Environment and Energy, Canada.

https://atrium.lib.uoguelph.ca/xmlui/bitstream/handle/10 214/15797/OME_guidelines_aquatsed_qual_prot93.pdf? sequence $=1 \&$ isAllowed $=y$.

Poerschmann J., Koschorreck M. \& Górecki T. 2017. Organic matter in sediment layers of an acidic mining lake as assessed by lipid analysis. Part II: Neutral lipids. Science of The Total Environment, 578: 219-227. http://dx.doi.org/10.1016/j.scitotenv.2016.01.116.

Portz L., Portantiolo R., Francisco C., Villate D., Bolivar D. \& Alcántara J. 2020. Assessment of Heavy Metals Pollution $(\mathrm{Hg}, \mathrm{Cr}, \mathrm{Cd}, \mathrm{Ni})$ in the Sediments of Mallorquin Lagoon - Barranquilla, Colombia. Journal of Coastal Research, 95(sp1): 158-162. https://doi.org/10.2112/SI95-031.1.

Ramírez J. \& Noreña J. 2004. Caracterización del sedimento de una laguna tropical rasa. Caldasia, 26(1): 173-184. https://revistas.unal.edu.co/index.php/cal/article/view/39 360

Rubio-Arias H., Mejía-Leyva P., Cortés-Palacios L., OchoaRivero J. \& De La Mora-Orozco C. 2018. Metales pesados en sedimentos de la Laguna de Bustillos, Chihuahua, México y comparación de agua regia y peróxido de hidrógeno como métodos de digestión. Investigación y Ciencia, 26(74): 39-47. https://www.redalyc.org/articulo.oa?id=67455945006.

Santana M. 2020. Evaluación del riesgo de contaminación por metales pesados en sedimentos de manglar en Ecuador. Tesis de Maestría, Universidad de Guayaquil. Ecuador. URI: http://repositorio.ug.edu.ec/handle/redug/51361.

Secretaria de Gobernación. 2002. Norma Oficial Mexicana NOM-021 RECNAT-2000, que establece las especificaciones para los estudios de fertilidad, salinidad y clasificación de suelos, su muestreo y análisis. SEMARNAP. Diario Oficial de la Federación (DOF), 31/12/2002

México. http://dof.gob.mx/nota_detalle.php?codigo $=717582 \&$ fec ha $=31 / 12 / 2002$
SEMARNAT (Secretaría de Medio Ambiente y Recursos Naturales). 2007. Norma Oficial Mexicana NOM-147SEMARNAT/SSA1-2004, Que establece criterios para determinar las concentraciones de remediación de suelos contaminados por arsénico, bario, berilio, cadmio, cromo hexavalente, mercurio, níquel, plata, plomo, selenio, talio y/o vanadio. Diario Oficial, viernes 2 de marzo de 2007: 1-69. México. http://www.profepa.gob.mx/innovaportal/file/1392/1/no m-147-semarnat_ssa1-2004.pdf.

Temporetti P., Antonuk L. \& Pedrozo, F. 2014. Características de los sedimentos de la Bahía Oriental del Lago Lácar afectado por la descarga de aguas residuales. Ecol. Austral, 24(3): 294-303. https://doi.org/10.25260/EA.14.24.3.0.6.

Tendaupenyu P. \& Magadza C. 2019. Enrichment and geoaccumulation of metals in the superficial sediments of Lake Chivero, Zimbabwe. Lakes \& Reservoirs, 24(3): 275-286. https://doi.org/10.1111//re.12282.

USEPA. 1993. Selecting Remediation Techniques for Contaminated Sediment, USEPA-823-B93-001. Office of Water (WH-585). USEPA (United States Environmental Protection Agency). https://nepis.epa.gov/Exe/ZyPDF.cgi/20003Q0N.PDF?D ockey $=20003$ Q0N.PDF.

USEPA. 1996. Method 3050B acid digestion of sediments, sludges, and soils. USEPA (United States Environmental Protection Agency). https://www.epa.gov/sites/production/files/201506/documents/epa-3050b.pdf.

Varol M. 2020. Spatio-temporal changes in surface water quality and sediment phosphorus content of a large reservoir in Turkey, Environmental Pollution, 259: 113860. https://doi.org/10.1016/j.envpol.2019.113860.

Villalba N. 2020. Determinación de la concentración de contaminantes en sedimentos superficiales y su relación con la estructura de comunidades bentónicas en la costa de Antioquia, caribe colombiano. Tesis de Magister, Universidad de Antioquia. Colombia. http://bibliotecadigital.udea.edu.co/handle/10495/15860

Villaseñor D. 2015. Fundamentos y procedimientos para análisis físico morfológicos de suelos. Universidad Técnica de Machala, Ecuador. http://repositorio.utmachala.edu.ec/bitstream/48000/106 90/1/FUNDAMENTOS\%20Y\%20PROCEDIMIENTOS .pdf

Xu F.-L., Yang C., He W., He Q.-S., Li Y.-L., Kang L., Liu W.-X., Xiong Y.-Q. \& Xing B. 2017. Bias and association of sediment organic matter source apportionment indicators: A case study in a eutrophic Lake Chaohu, China. Science of The Total Environment, 581-582:

874-884. https://doi.org/10.1016/j.scitotenv.2017.01.037.

Zagal E. \& Sadzawka A. 2007. Protocolo de métodos de análisis para suelos y lodos. Universidad de Concepción. Chile.

http://www.sag.cl/sites/default/files/METODOS_LODO S_SUELOS.pdf. 
Tabla 3. Referencia para límites permisibles para metales en sedimentos establecidos por normas ambientales de EEUU, México y Canadá (en mg/Kg).

\begin{tabular}{|c|c|c|c|c|c|c|}
\hline NORMAS & & $\mathrm{Pb}$ & $\mathrm{Cu}$ & $\mathrm{Fe}$ & $\mathrm{Mn}$ & $\mathrm{Zn}$ \\
\hline \multirow[t]{3}{*}{ USEPA-823-B93-001 (USEPA, 1993) } & NP & $<40$ & $<25$ & $<17000$ & & $<90$ \\
\hline & MP & $40-60$ & $25-50$ & $1700-25000$ & & $90-200$ \\
\hline & $\mathrm{HP}$ & $>60$ & $>50$ & $>25000$ & & $>200$ \\
\hline $\begin{array}{l}\text { NOM-147-SEMARNAT/SSA1-2004 } \\
\text { (SEMARNAT, 2007) }\end{array}$ & & 400 & & & & \\
\hline Canadian Sediment Quality Guidelines for the & ISQG & 35 & 35.7 & & & 123 \\
\hline Protection of Aquatic Life (CCME, 2001) & PEL & 91.3 & 197 & & & 315 \\
\hline \multirow[t]{2}{*}{ Norma Ontario (Persaud et al., 1993) } & LEL & 31 & 16 & 20000 & 460 & 120 \\
\hline & SEL & 250 & 110 & 40000 & 1100 & 820 \\
\hline NOAA (NOAA, 2008) & & $4-17$ & $10-25$ & $9900-18000$ & 400 & $7-38$ \\
\hline
\end{tabular}

Leyenda: NP: No contaminado; MP: Moderadamente contaminado; HP: Altamente contaminado; ISQG: Guía provisional de calidad del sedimento; PEL: Nivel de efecto probable; LEL: Nivel de efecto más bajo; SEL: Nivel de efecto severo.

Tabla 4. Composición físico-química de los sedimentos del humedal Laguna Bella.

\begin{tabular}{|c|c|c|c|c|c|c|c|c|}
\hline \multirow[t]{2}{*}{$\overline{\text { Parámetros }}$} & \multicolumn{6}{|c|}{ Puntos de muestreo } & \multicolumn{2}{|c|}{ TOTAL } \\
\hline & $\begin{array}{c}P_{1} \\
\text { Media } \\
\pm \mathrm{EE}\end{array}$ & $\begin{array}{c}\mathbf{P}_{2} \\
\text { Media } \\
\pm \mathbf{E E}\end{array}$ & $\begin{array}{c}P_{3} \\
\text { Media } \\
\pm \mathrm{EE}\end{array}$ & $\begin{array}{c}\mathbf{P}_{4} \\
\text { Media } \\
\pm \mathbf{E E}\end{array}$ & $\begin{array}{c}P_{5} \\
\text { Media } \\
\pm \text { EE }\end{array}$ & $\begin{array}{c}\mathbf{P}_{6} \\
\text { Media } \\
\pm \mathrm{EE}\end{array}$ & $\begin{array}{c}\text { Media } \\
\pm \text { EE }\end{array}$ & $\begin{array}{l}\text { CV } \\
(\%)\end{array}$ \\
\hline Humedad (\%) & $\begin{array}{r}63.99 \\
\pm 1.45\end{array}$ & $\begin{array}{r}63.89 \\
\pm 1.25\end{array}$ & $\begin{array}{l}60.61 \\
\pm 2.46\end{array}$ & $\begin{array}{r}64.09 \\
\pm 1.86\end{array}$ & $\begin{array}{r}59.70 \\
\pm 1.72\end{array}$ & $\begin{array}{r}67.43 \\
\pm 0.67\end{array}$ & $\begin{array}{r}63.28 \\
\pm 0.84\end{array}$ & 5.63 \\
\hline $\begin{array}{l}\text { Materia } \\
\text { orgánica (\%) }\end{array}$ & $\begin{array}{l}12.05 \\
\pm 0.06\end{array}$ & $\begin{array}{r}11.67 \\
\pm 0.07\end{array}$ & $\begin{array}{l}11.74 \\
\pm 0.05\end{array}$ & $\begin{array}{r}11.96 \\
\pm 0.06\end{array}$ & $\begin{array}{r}11.26 \\
\pm 0.02\end{array}$ & $\begin{array}{l}11.96 \\
\pm 0.06\end{array}$ & $\begin{array}{l}11.77 \\
\pm 0.07\end{array}$ & 2.40 \\
\hline $\mathrm{pH}$ & $\begin{array}{l}7.75 \\
\pm 0.03\end{array}$ & $\begin{array}{l}7.85 \\
\pm 0.01\end{array}$ & $\begin{array}{l}7.89 \\
\pm 0.01\end{array}$ & $\begin{array}{c}7.84 \\
\pm 0.02\end{array}$ & $\begin{array}{c}7.98 \\
\pm 0.02\end{array}$ & $\begin{array}{c}7.67 \\
\pm 0.03\end{array}$ & $\begin{array}{c}7.83 \\
\pm 0.03\end{array}$ & 1.34 \\
\hline $\begin{array}{l}\text { Conductividad eléctrica } \\
(\mu \mathrm{S} / \mathrm{cm})\end{array}$ & $\begin{array}{l}269.72 \\
\pm 7.00\end{array}$ & $\begin{array}{l}236.40 \\
\pm 1.23\end{array}$ & $\begin{array}{l}223.39 \\
\pm 1.90\end{array}$ & $\begin{array}{l}215.48 \\
\pm 1.34\end{array}$ & $\begin{array}{l}188.14 \\
\pm 1.26\end{array}$ & $\begin{array}{l}244.67 \\
\pm 0.54\end{array}$ & $\begin{array}{l}229.63 \\
\pm 0.54\end{array}$ & 0.38 \\
\hline $\begin{array}{l}\text { Densidad } \\
\left(\mathrm{g} / \mathrm{cm}^{3}\right)\end{array}$ & $\begin{array}{l}2.45 \\
\pm 0.05\end{array}$ & $\begin{array}{l}2.55 \\
\pm 0.01\end{array}$ & $\begin{array}{c}2.63 \\
\pm 0.14\end{array}$ & $\begin{array}{c}2.60 \\
\pm 0.03\end{array}$ & $\begin{array}{c}2.65 \\
\pm 0.05\end{array}$ & $\begin{array}{c}2.52 \\
\pm 0.01\end{array}$ & $\begin{array}{c}2.57 \\
\pm 0.03\end{array}$ & 4.66 \\
\hline $\begin{array}{l}\text { Nitrógeno } \\
(\%)\end{array}$ & $\begin{array}{l}0.204 \\
\pm 0.01\end{array}$ & $\begin{array}{l}0.206 \\
\pm 0.02\end{array}$ & $\begin{array}{l}0.212 \\
\pm 0.00\end{array}$ & $\begin{array}{l}0.282 \\
\pm 0.02\end{array}$ & $\begin{array}{l}0.186 \\
\pm 0.01\end{array}$ & $\begin{array}{l}0.223 \\
\pm 0.01\end{array}$ & $\begin{array}{c}0.22 \\
\pm 0.01\end{array}$ & 17.97 \\
\hline $\begin{array}{l}\text { Fósforo } \\
(\mathrm{mg} / \mathrm{Kg})\end{array}$ & $\begin{array}{l}48.46 \\
\pm 0.24 \\
\end{array}$ & $\begin{array}{r}42.89 \\
\pm 0.44 \\
\end{array}$ & $\begin{array}{l}31.02 \\
\pm 0.47 \\
\end{array}$ & $\begin{array}{r}32.39 \\
\pm 0.51 \\
\end{array}$ & $\begin{array}{r}32.69 \\
\pm 0.66\end{array}$ & $\begin{array}{l}25.08 \\
\pm 0.30 \\
\end{array}$ & $\begin{array}{l}35.42 \\
\pm 1.64 \\
\end{array}$ & 22.72 \\
\hline
\end{tabular}

Tabla 5. Contenido de elementos metálicos en los sedimentos del humedal Laguna Bella.

\begin{tabular}{|c|c|c|c|c|c|c|c|c|}
\hline \multirow{2}{*}{ 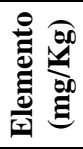 } & \multicolumn{6}{|c|}{ Puntos de muestreo } & \multicolumn{2}{|c|}{ Total } \\
\hline & $\begin{array}{c}P_{1} \\
\text { Media } \\
\pm \text { EE }\end{array}$ & $\begin{array}{c}\mathrm{P}_{2} \\
\text { Media } \\
\pm \mathrm{EE}\end{array}$ & $\begin{array}{c}P_{3} \\
\text { Media } \\
\pm \mathrm{EE}\end{array}$ & $\begin{array}{c}P_{4} \\
\text { Media } \\
\pm \text { EE }\end{array}$ & $\begin{array}{c}P_{5} \\
\text { Media } \\
\pm \text { EE }\end{array}$ & $\begin{array}{c}\text { P6 } \\
\text { Media } \\
\pm \text { EE }\end{array}$ & $\begin{array}{l}\text { Media } \\
\pm \text { EE }\end{array}$ & $\begin{array}{l}\text { CV } \\
(\%)\end{array}$ \\
\hline $\mathrm{Pb}$ & 62.42 & 63.54 & 59.62 & 59.33 & 59.33 & 56.85 & 59.78 & 5.01 \\
\hline & \pm 1.86 & \pm 1.29 & \pm 0.75 & \pm 0.20 & \pm 0.20 & \pm 0.15 & \pm 0.71 & \\
\hline $\mathrm{Cu}$ & $\begin{array}{r}34.60 \\
\pm 0.34\end{array}$ & $\begin{array}{l}35.71 \\
\pm 0.47\end{array}$ & $\begin{array}{r}35.29 \\
\pm 0.21\end{array}$ & $\begin{array}{l}35.60 \\
\pm 0.19\end{array}$ & $\begin{array}{r}35.60 \\
\pm 0.19\end{array}$ & $\begin{array}{l}35.22 \\
\pm 0.10\end{array}$ & $\begin{array}{l}35.24 \\
\pm 0.13\end{array}$ & 1.53 \\
\hline $\mathrm{Fe}$ & $\begin{array}{c}34900.58 \\
\pm 348.50\end{array}$ & $\begin{array}{c}37416.99 \\
\pm 837.55\end{array}$ & $\begin{array}{c}42189.80 \\
\pm 816.74\end{array}$ & $\begin{array}{c}42290.82 \\
\pm 407.51\end{array}$ & $\begin{array}{c}42290.82 \\
\pm 407.51\end{array}$ & $\begin{array}{r}44759.46 \\
\pm 1077.81\end{array}$ & $\begin{array}{c}40430.57 \\
\pm 844.83\end{array}$ & 8.87 \\
\hline $\mathrm{Mn}$ & $\begin{array}{l}678.75 \\
\pm 2.81\end{array}$ & $\begin{array}{l}806.39 \\
\pm 2.20\end{array}$ & $\begin{array}{r}864.31 \\
\pm 0.71\end{array}$ & $\begin{array}{l}918.29 \\
\pm 1.36\end{array}$ & $\begin{array}{l}918.29 \\
\pm 1.36\end{array}$ & $\begin{array}{l}892.93 \\
\pm 1.26\end{array}$ & $\begin{array}{r}839.10 \\
\pm 19.27\end{array}$ & 9.74 \\
\hline $\mathrm{Zn}$ & $\begin{array}{c}69.43 \\
\pm 0.88\end{array}$ & $\begin{array}{r}72.41 \\
\pm 1.31\end{array}$ & $\begin{array}{l}83.62 \\
\pm 1.64\end{array}$ & $\begin{array}{c}86.14 \\
\pm 1.68\end{array}$ & $\begin{array}{r}86.14 \\
\pm 1.68\end{array}$ & $\begin{array}{r}84.48 \\
\pm 0.83\end{array}$ & $\begin{array}{l}79.21 \\
\pm 1.67\end{array}$ & 8.92 \\
\hline $\mathrm{K}$ & $\begin{array}{c}2326.73 \\
\pm 6.97 \\
\end{array}$ & $\begin{array}{c}2582.69 \\
\pm 12.46 \\
\end{array}$ & $\begin{array}{c}4181.27 \\
\pm 6.41 \\
\end{array}$ & $\begin{array}{c}4896.05 \\
\pm 10.51 \\
\end{array}$ & $\begin{array}{c}4896.05 \\
\pm 10.51 \\
\end{array}$ & $\begin{array}{l}5138.28 \\
\pm 4.29 \\
\end{array}$ & $\begin{array}{r}4052.64 \\
\pm 285.98 \\
\end{array}$ & 29.94 \\
\hline
\end{tabular}

\footnotetext{
${ }^{1}$ Universidad Nacional Agraria de la Selva. Carretera Central km. 1.21, Tingo María / Perú. antoniozav04@hotmail.com.

${ }^{2}$ Universidad Nacional de Cañete. Jr. San Agustín 124, San Vicente de Cañete / Cañete / Lima / Perú. Autor de correspondencia: mnique@undc.edu.pe.

${ }^{3}$ Universidad Nacional Agraria de la Selva. Carretera Central km. 1.21, Tingo María / Perú. joselevano@hotmail.com.
} 